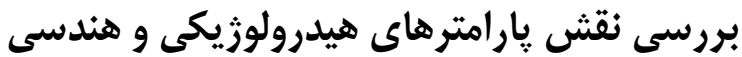

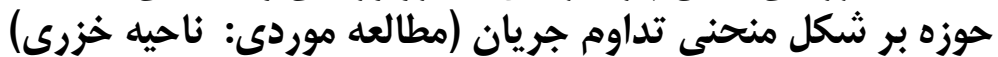

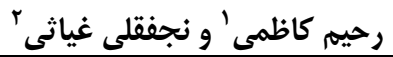

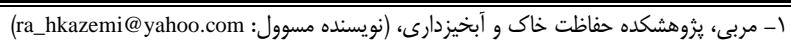

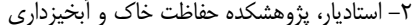

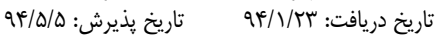

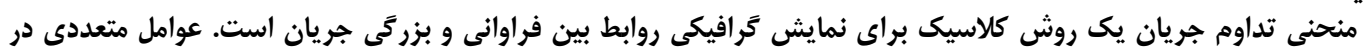

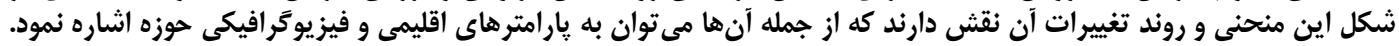

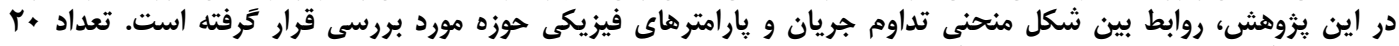

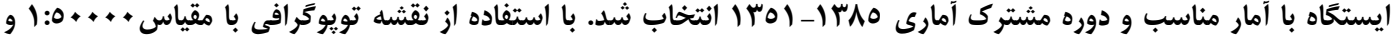

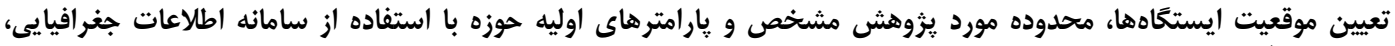

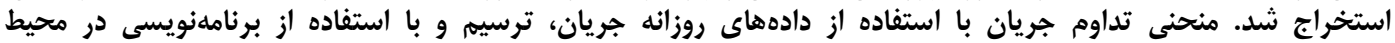

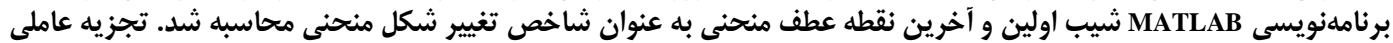

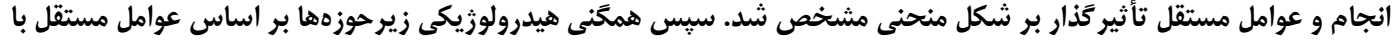

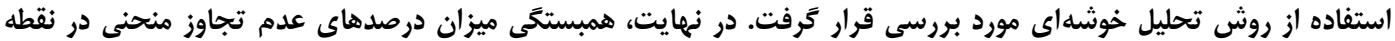

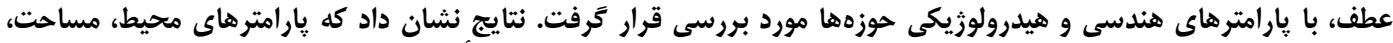

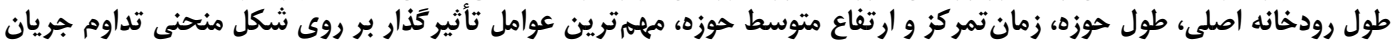

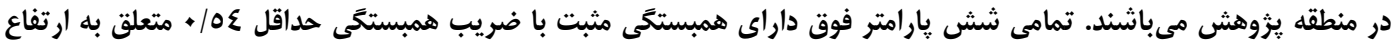

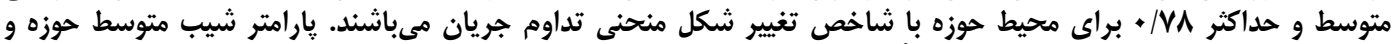

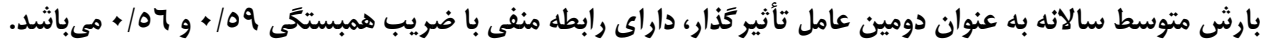

وازمهاى كليدى: حوزه خزر، شاخص تغييرشكل، مناطق همَّن، منحنى تداوم جريان، نقطه عطف منحنى

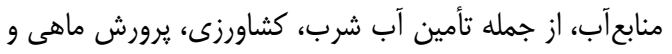

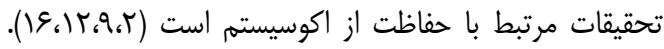

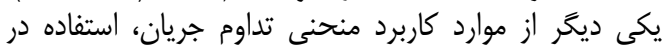

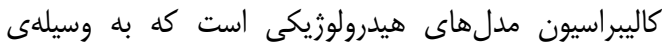

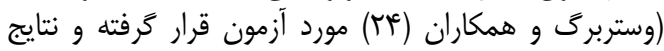

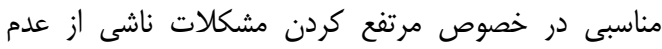

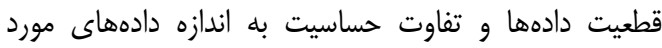

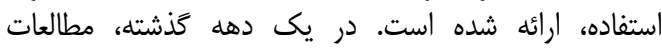

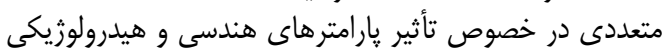

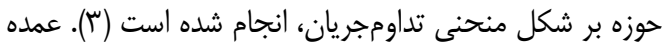

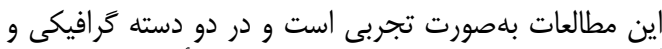

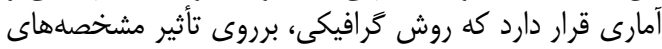

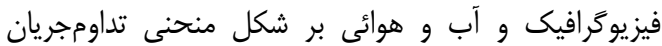

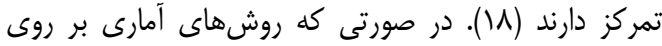

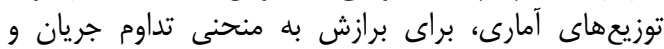

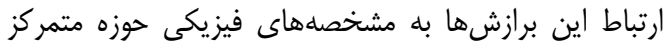

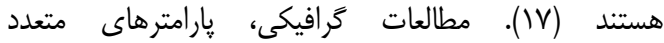

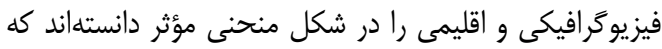

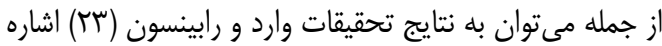

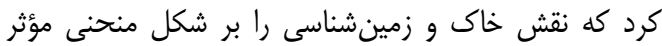

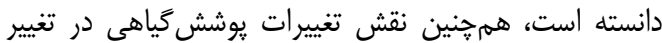
شكل منحنى تداوم جريان به وسيله لان و همكاران (هات به به به

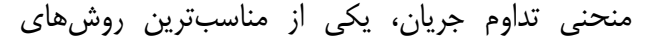

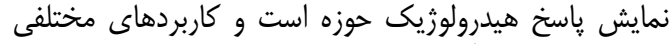

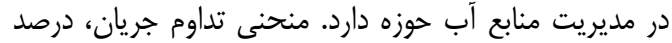

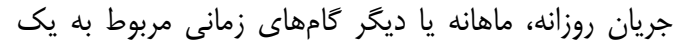

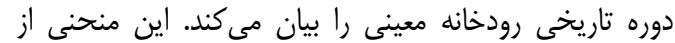

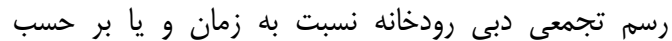
احتمال وقوع بهدست مى آيد و مكرراً براى مقايسه رئ رزيم

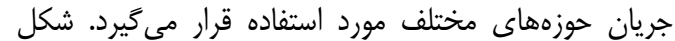

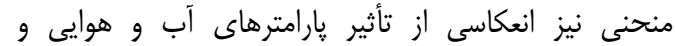

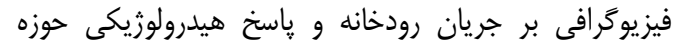

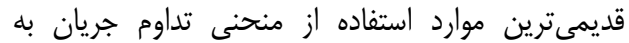

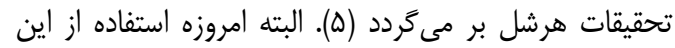

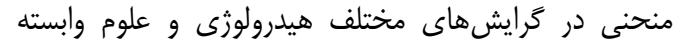

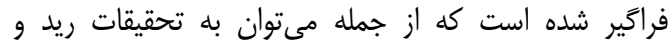

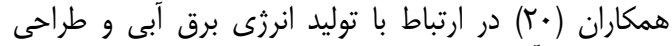

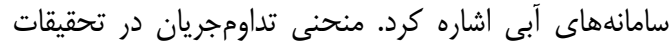

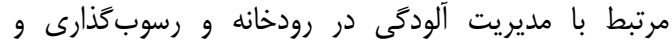

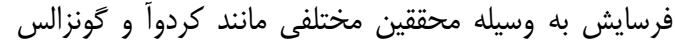

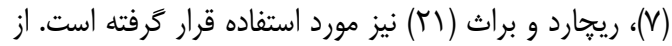
ديخر موارد كاربرد اين منحنى، استفاده آن در برناماهريزي 
هيدرولوزيكى و فيزيوكرافى، موجب دستيابى بهاطالاعاتى

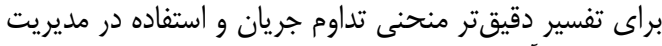
بهينه منابع آب حوزه خواهد شد تدر.

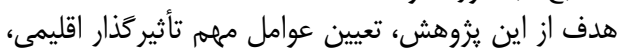

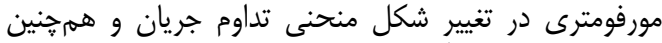
بررسى روابط مابين آنها مىباشد.

\section{مواد و روشها - مورديا} منطقه مورد يزوهش موري

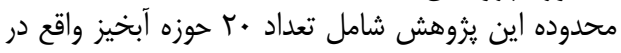

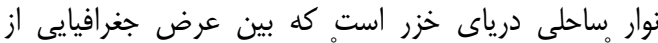

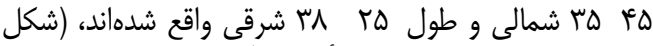

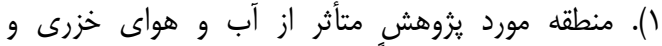

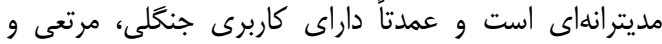

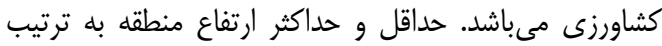

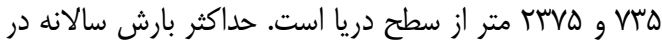

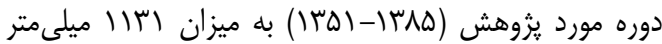

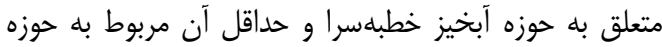

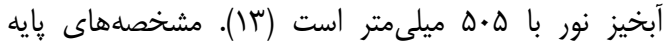

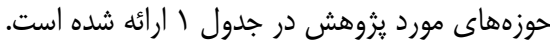

اثبات رسيده است. تحقيقات زانح و همكاران (كه) در حوزه

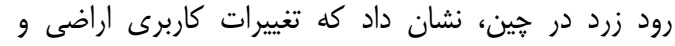

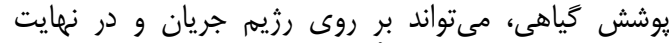

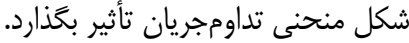

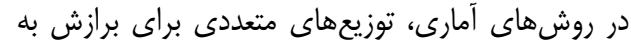

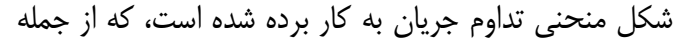

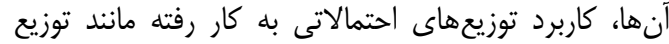

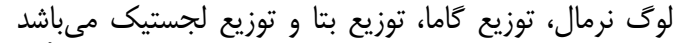

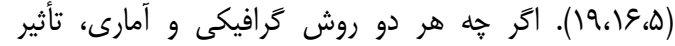

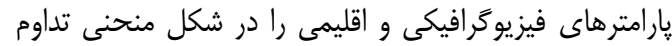

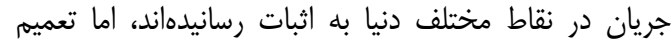
نتايج از نقطهاى به نقطه ديخر به دئ دليل تنوع عوامل تأثير كذار،

مفيد نخواهد بود (؟).

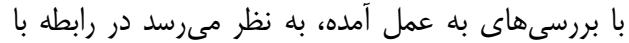

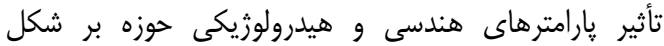

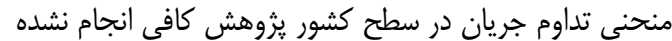

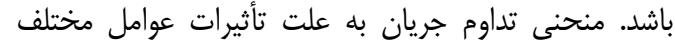

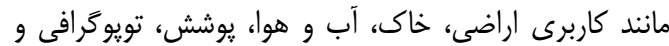

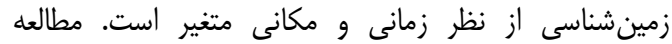

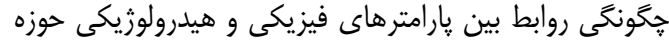

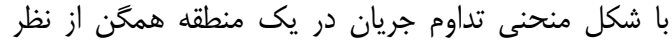

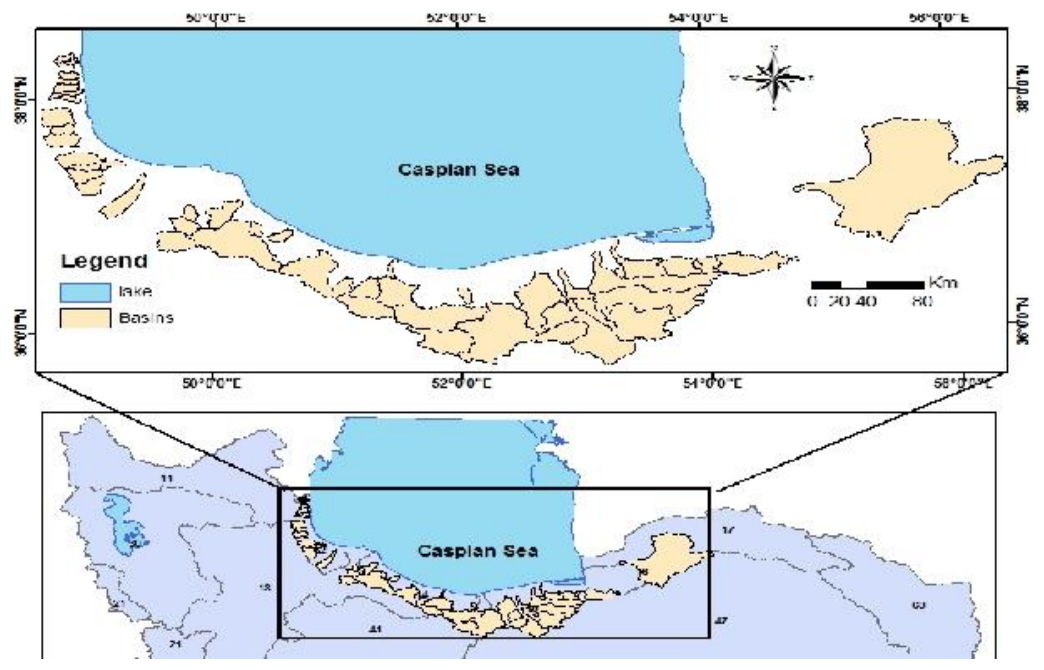

شكل 1- منطقه مورد يزوهش

Figure 1. Location of the study area

MATLAB

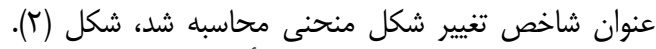

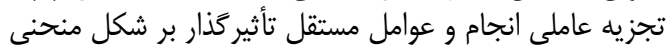

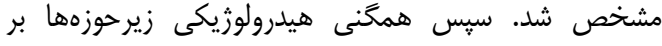

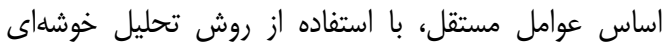

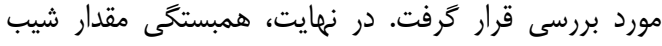

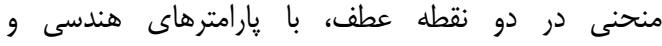

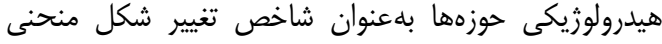
تداوم جريان مورد بررسى قرار كرفت.

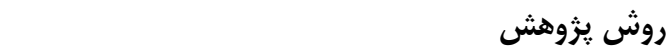

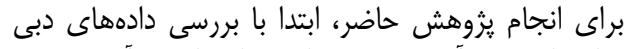

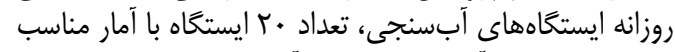

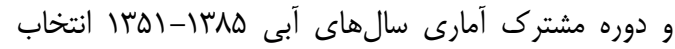

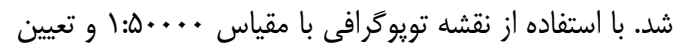

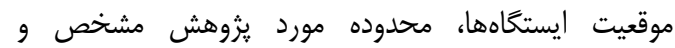

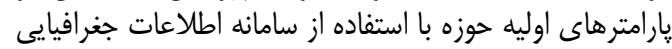

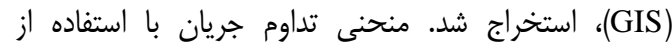

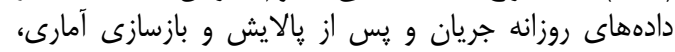
ترسيم و با استفاده از برنامهنويسى در محيط برنان بانه نويسى آناري 
Table 1. The characteristics of the studied catchments

جدول 1- مشخصههاى حوزههاى مورد يثوهش

\begin{tabular}{|c|c|c|c|c|c|c|}
\hline $\begin{array}{c}\text { بارش متوسط سالانه } \\
\text { (mm) }\end{array}$ & مساحت (Km²) & الرتفاع متوسط (m) & عرض جغرافيايى & طول جغرافيايى & كد ايستخاه هيدرومترى & نام حوزه \\
\hline $9 \wedge$. & $T V \cdot r$ & T.F & TE,TT & $\Delta H_{6}$. & $I T-\cdot T Q$ & ريخ جشمه تجن \\
\hline SQV & Irrq & $r \cdot 90$ & 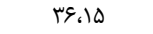 & 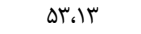 & $17+.19$ & سليمان تنكه-تجن \\
\hline qva & TQT & $|A M|$ & rg, L & QT.DT & $\| f-\cdots D$ & شير كاه (كسيليان) \\
\hline 971 & frye & 1994 & (9.19 & Dr.tA & $\mid f=.11$ & بابلرود (قران طالار) \\
\hline$\Delta \cdot \Delta$ & 1111 & TVYT & (49.1) & Qr.II & $10-\cdot 10$ & نور \\
\hline ع Q D D & mati & rq19 & ع & DT.TT & $10_{-+} \mid V$ & كره سنَ \\
\hline 909 & 91 & 1990 & 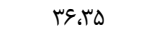 & $\Delta T \cdot \Delta F$ & $19-. . r$ & لاويج \\
\hline DFf & IETT & rmis & r9.r. & DI.T. & $\left|\varepsilon_{-\cdot r}\right|$ & حالوس \\
\hline STD & 191 & r.r. & q q. & DI..V V & سז.--4| & سردابرود ( كلاردشت) \\
\hline gr. & VVT & rFi. & 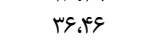 & $\Delta \cdot \Delta \Delta \Delta$ & $|9-\cdot 4|$ & جشمه كيله ( هراتبر) \\
\hline 1.19 & 99 & WASE & rV.Qq & $0.6 \mathrm{~V}$ & $19-.09$ & "سموش (هراتبر) \\
\hline FTV & זسז & iff. & سه، & 49.41 & $I V-\cdot F \Delta$ & توتكابن \\
\hline VTV & TrA & שוזו & $r V_{6} \cdot$. & +9.40 & $\left|V_{-} \cdot \Delta\right|$ & زيلكي (شهر بيجار) \\
\hline $1 \cdot 1$ & $I T V$ & $V \pi \Delta$ & rV. 1 & $k 9.50$ & $\mid V-\cdot \Delta \Delta$ & ديشام (ياشاكى) \\
\hline 111. & ITD & wat & TV.TY & $<q, 0$ & $11-.19$ & رودبارسر --هاف رود \\
\hline $1 \cdot v^{c}$ & TET & $10 \cdot F^{c}$ & TV.TY & $+q \cdot \cdot \Delta$ & $|\Lambda-\cdot r|$ & شفارود (يونل) \\
\hline$\Delta F \Delta$ & $\Delta \wedge \Delta$ & 19rq & TV.TA & $F \Lambda_{0} F^{*} \wedge$ & $1 \Lambda_{-}+r q$ & كر كانرود (گر كانرود) \\
\hline aft & 1919 & $|Q \Delta|$ & 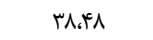 & $F \wedge_{\odot \Delta \Lambda}$ & $M$ & شير آباد \\
\hline 1.10 & $v \cdot$ & Ir.r & אוז.TI & $\left|\wedge \wedge_{(D}\right|$ & $1 \Lambda-. r q$ & خوبر (بالا محله) \\
\hline וTו & 99 & 119. & $r \Lambda_{\bullet} \cdot r$ & FANG & $\mid \Lambda_{-} \cdot \Delta \Delta$ & خطبه سرا \\
\hline
\end{tabular}

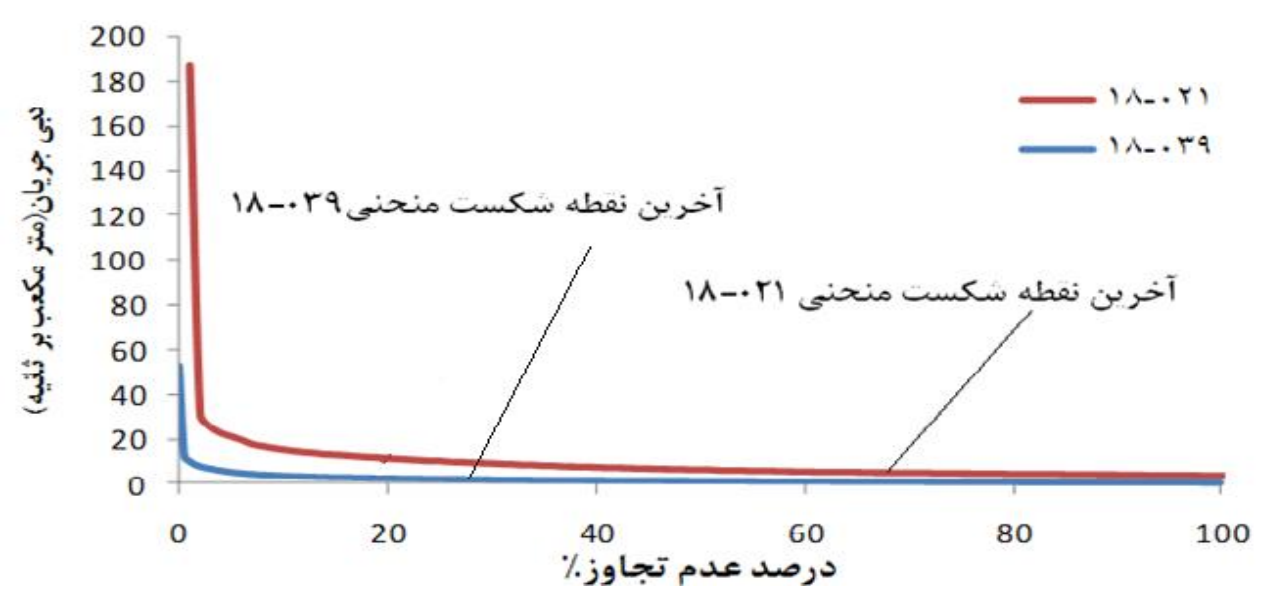

شكل r- منحنى تداوم جريان دو حوزه شفا رود و حوبر بالامحله با مشخصات هندسى و هيدرولوزيكى مختلف

Figure 2. Flow duration curve of Shafa Rood and Chobar Balamahalle catchments with different characteristics

به منظور به حداكثر رساندن واريانس بارهاى هر يكى از

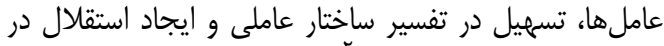

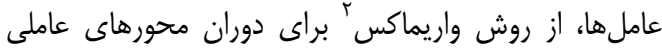

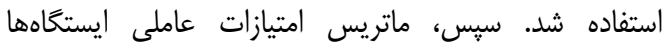

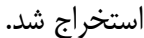
براى خارج كردن متغيرهاى غيرضرورى، از ماتريس

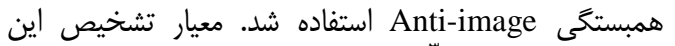

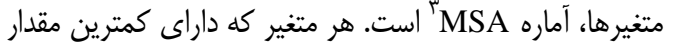

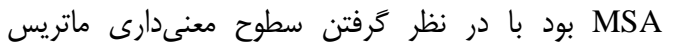

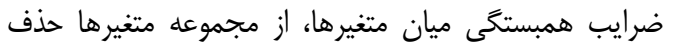

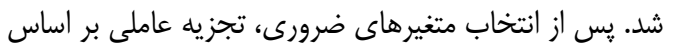

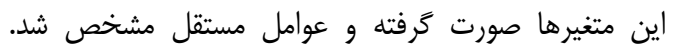
سبس همخَنى هيدرولوزيكى زيرحوزهها نيز بر اساس عوامل
تجزيه و تحليل عاملى ترو ترو در اين يروهش تجزيه و تحليه تحليل عاملى به به روش (PCA)

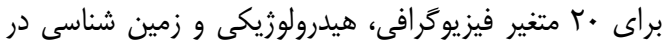

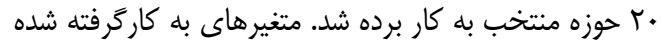

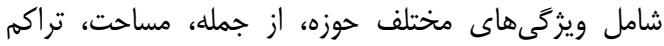

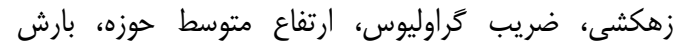

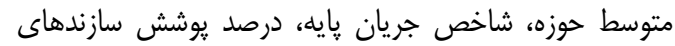

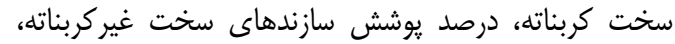

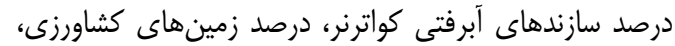

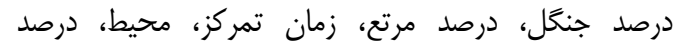

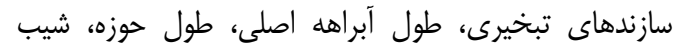

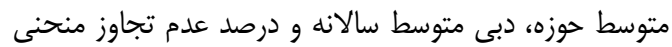
در آخرين نقطه عطف منحنى مى دباشند.

\footnotetext{
1- Principal Component Analysis

2- Varimax Rotation

3- Measure of Sampling Adequacy
} 


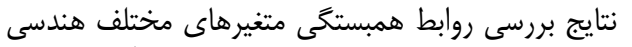

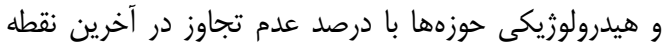

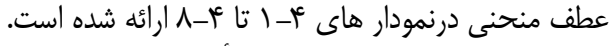

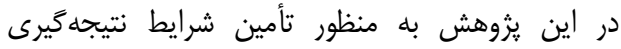

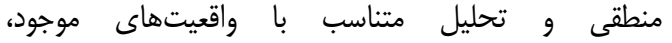

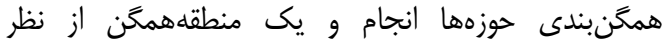

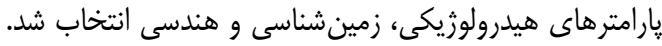

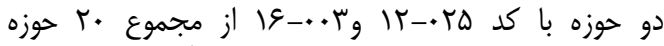

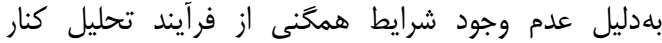

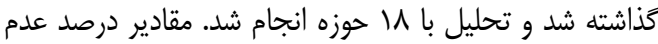

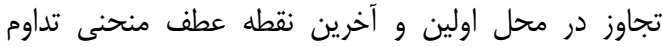

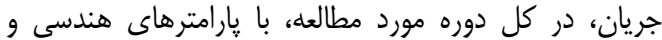

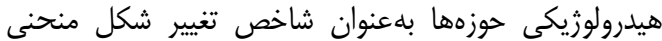

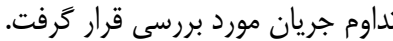

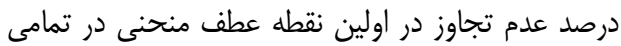

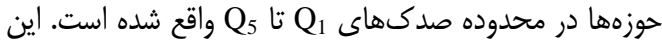

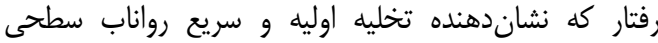

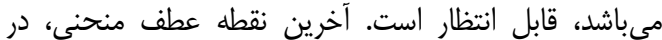

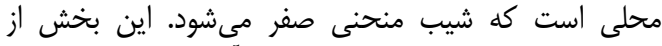

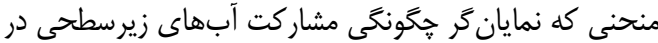

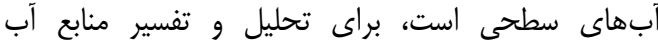

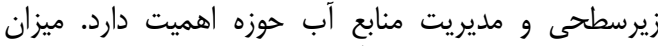

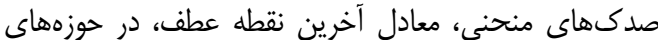
منطقه يثروهش، بين Q نا Q
مستقل، با استفاده از روش تحليل خوشهاى مورد بررسى قرار

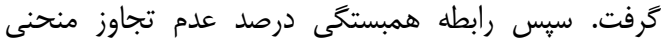

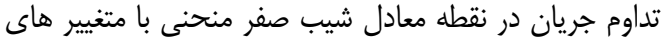
هندسى و هيدرولوزيكى حوزهها استخراج و مورد تحليل معليل قرار

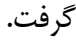

\section{نتايج و بحث}

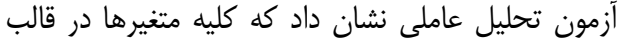

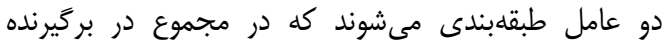

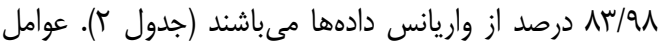

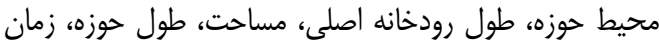

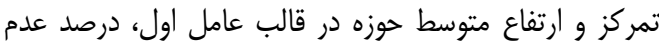

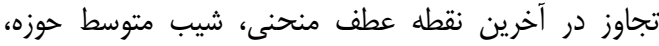

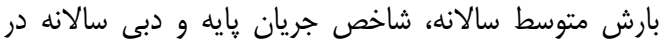

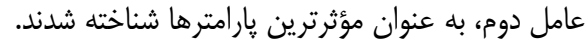

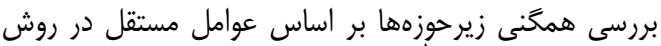

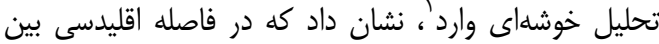

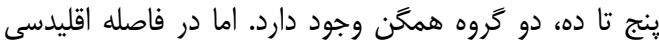

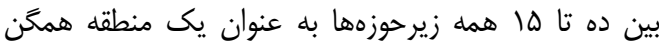

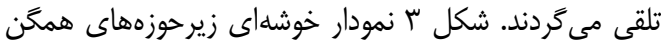

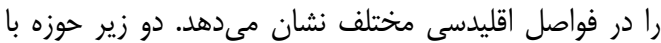

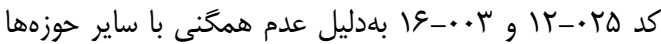

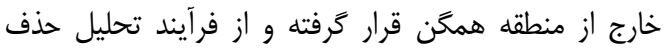

جدول r- كل درصد از واريانس توضيح داده شده بر اساس عوامل انتخابى درآزمون تحليل عاملى Table 2. Total percentage of variance explained based on selected factors in factor analysis

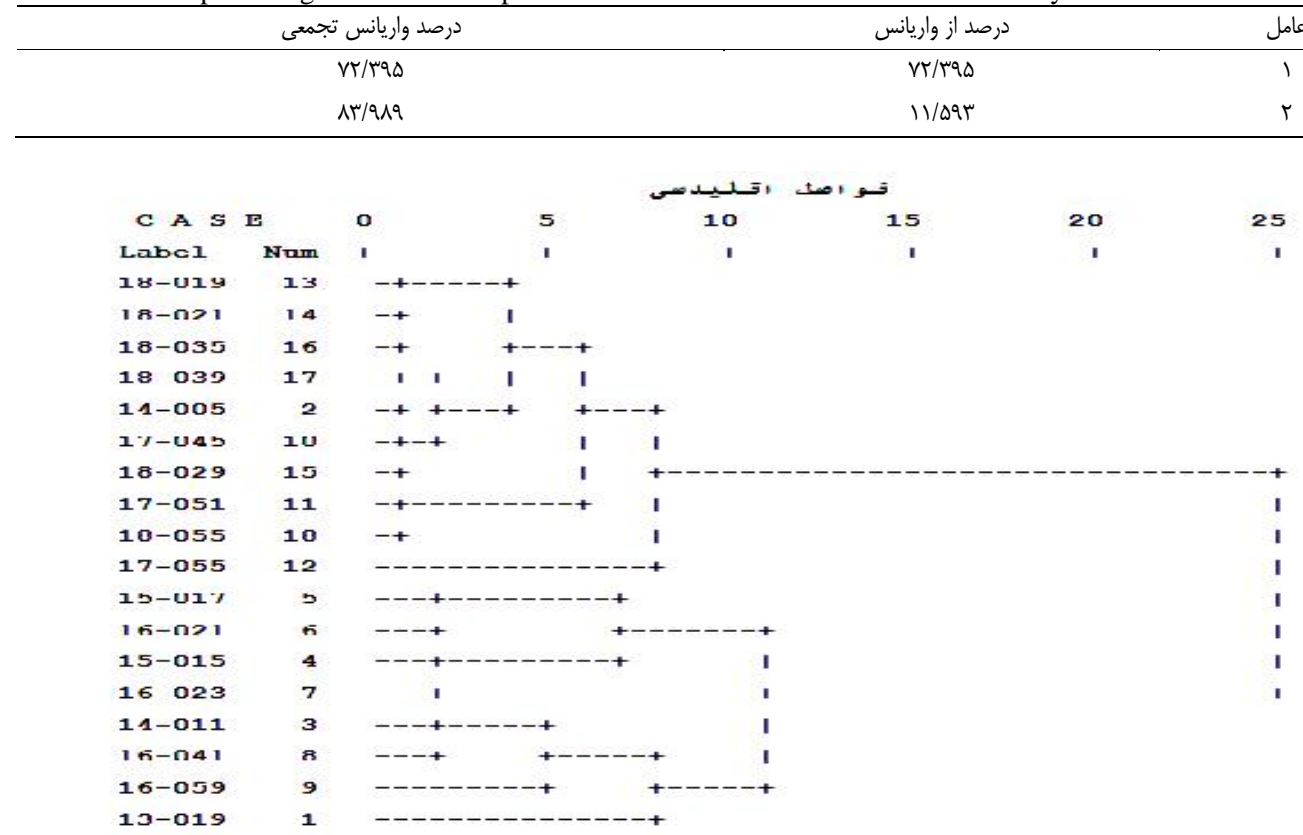

شكل سـ- نمودار خوشهاى زيرحوزهاى همخَن بر اساس عوامل مستقل

Figure 3. Dendrograom of homogeny catchments based of independent factors 


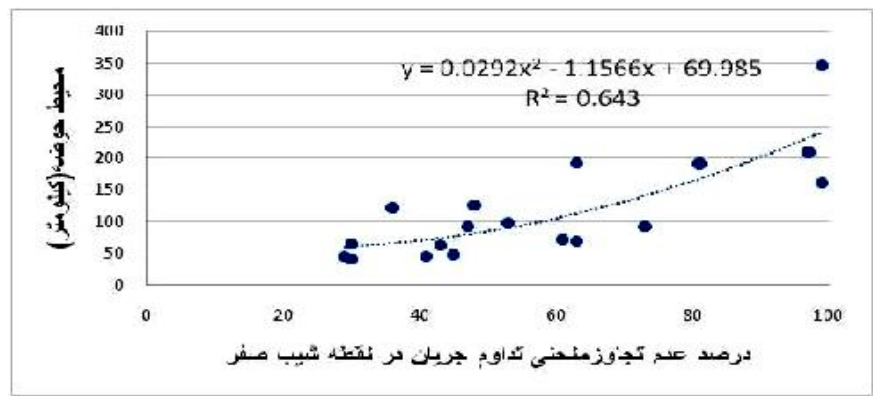

شكل ع ا-1- رابطه درصد عدم تجاوز در نقطه شيب صفر منحنى با محيط حوزهها

Figure 4-1. Relationship between exceedance value at the point of zero slope with catchment perimeter

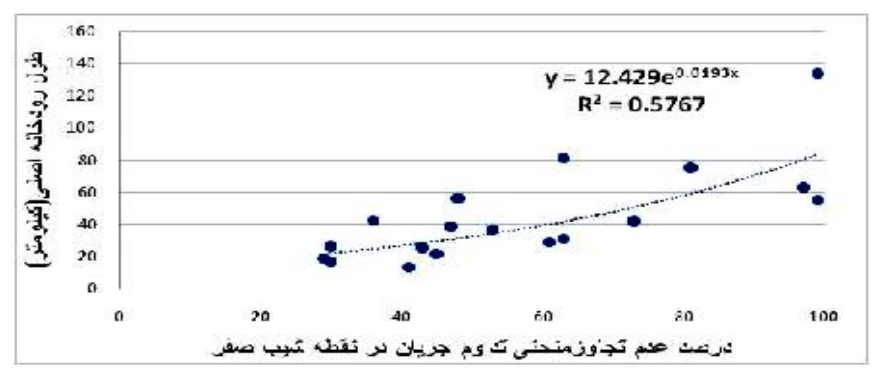

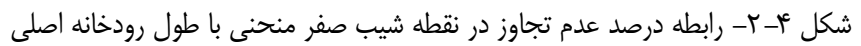

Figure 4-2. Relationship between exceedance value at the point of zero slope with the main river length

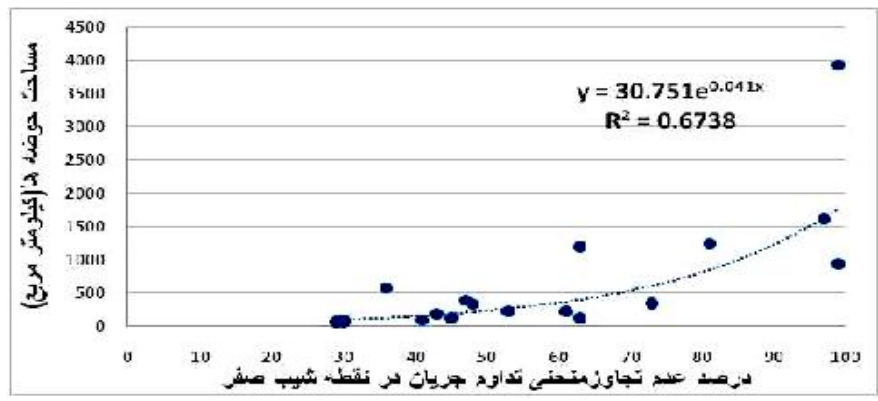

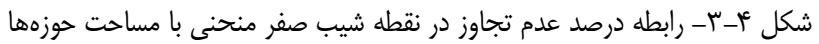

Figure 4-3. Relationship between exceedance value at the point of zero slope with the Catchment area

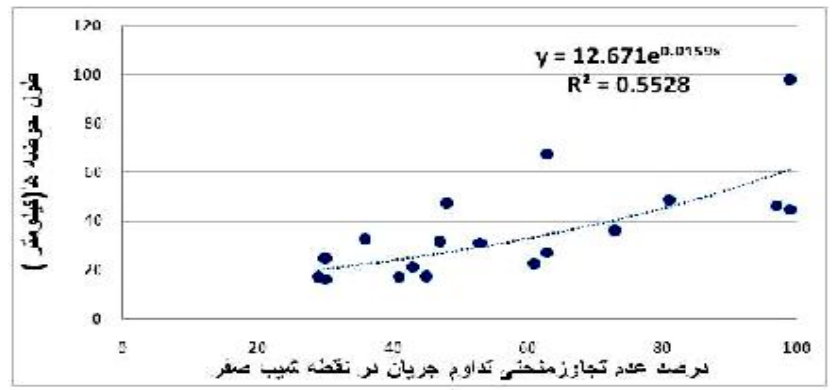

شكل f-f-f- رابطه درصد عدم تجاوز در نقطه شيب صفر منحنى با طول حوزهها

Figure 4-4. Relationship between exceedance value at the point of zero slope with the Catchment length 


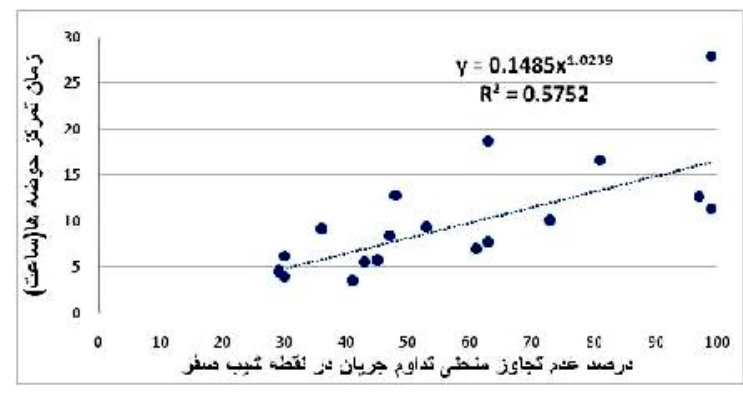

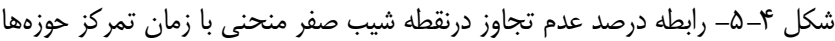

Figure 4-5. Relationship between exceedance value at the point of zero slope with the time of concentration

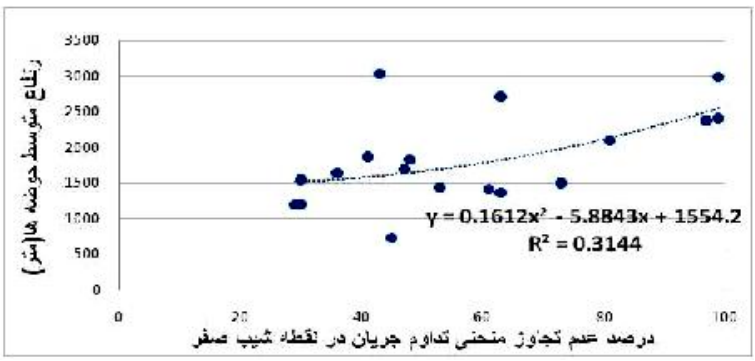

شكل ع ا-צ- رابطه درصد عدم تجاوز در نقطه شيب صفر منحنى با ارتفاع متوسط حوزهنها

Figure 4-6. Relationship between exceedance value at the point of zero slope with the average height of catchments

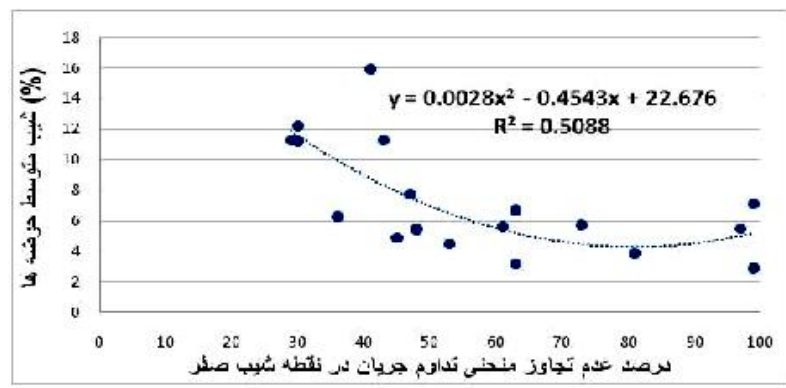

شكل ع-Y- ر- رابطه درصد عدم تجاوز در نقطه شيب صفر منحنى با شيب متوسط حوزهها

Figure 4-7. Relationship between exceedance value at the point of zero slope with the average slope of catchments

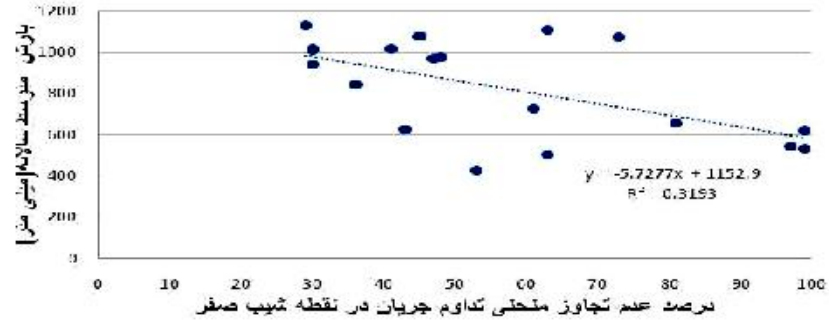

شكل ع-1-1 رابطه درصد عدم تجاوز درنقطه شيب صفر منحنى با بارش متوسط سالانه حوزها

Figure 4-8. Relationship between exceedance value at the point of zero slope with the average annual rainfall of the catchments 
جريان گايه و دبى متوسط سالانه به دليل ارتباط ذاتى با تريا

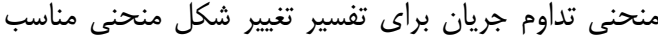

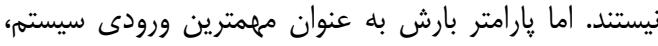

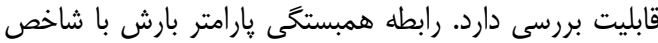

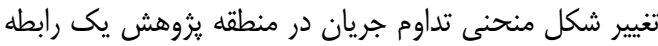

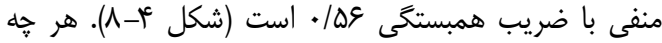

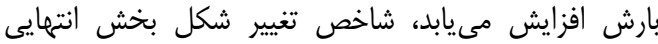

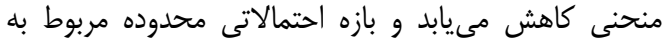

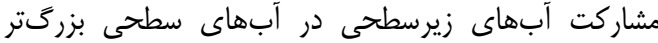

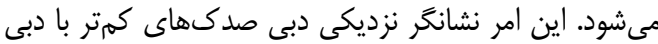

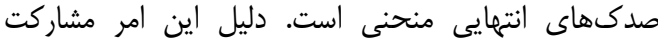
بيشتر جريانهاى زيرسطحى و تأخيرى در تأمين جرئ دانيان

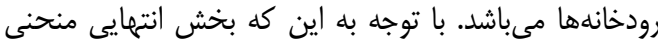

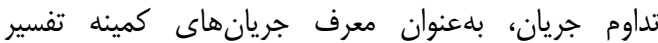

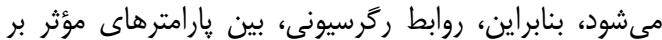

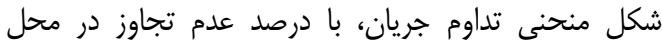

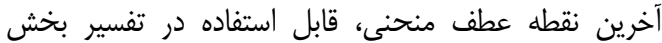

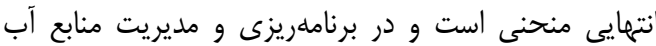

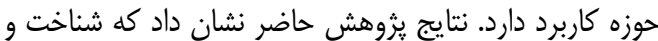

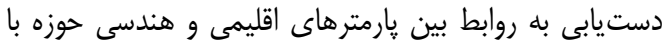

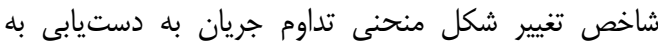

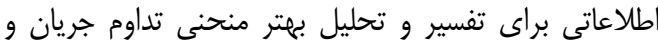

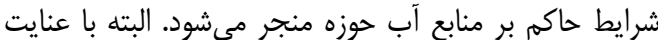

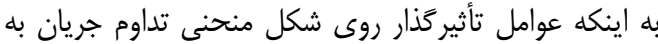

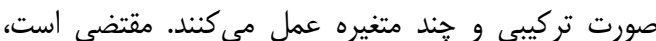

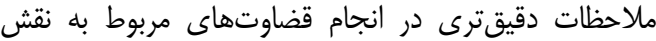

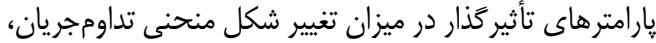

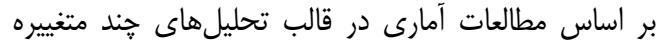
صورت يذيرد.

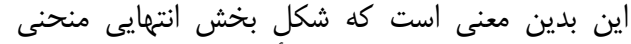

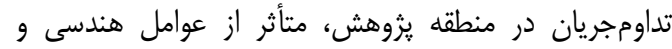

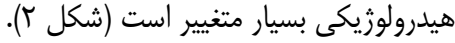

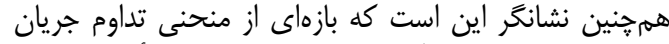

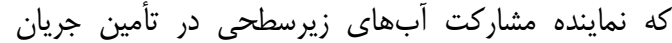

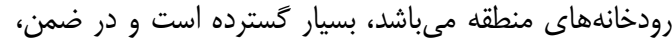

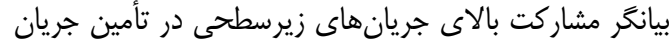

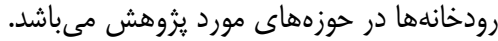

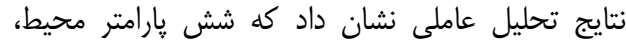

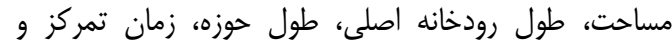

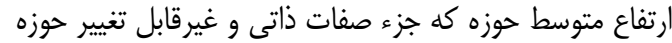

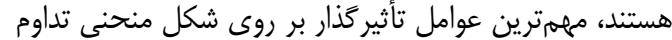

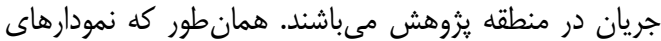

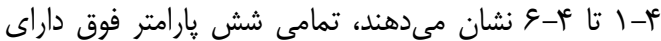

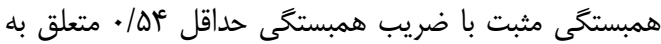

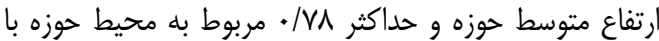

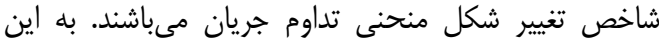

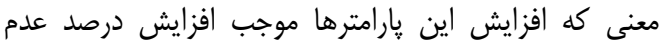

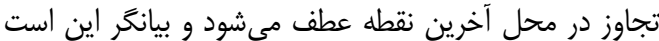

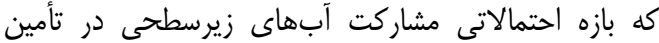

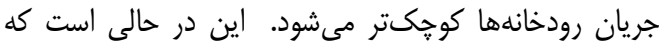

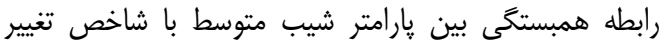

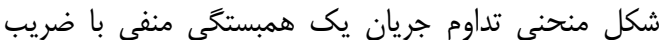

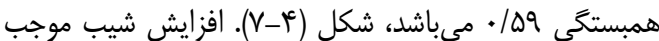

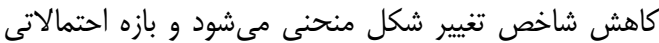

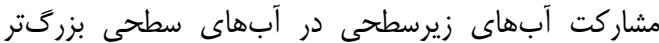

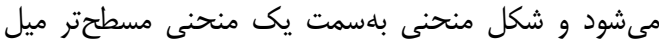

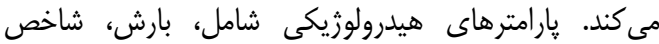

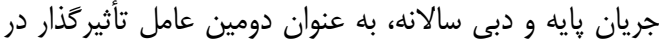

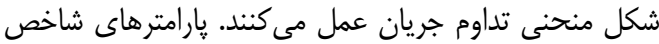

1. Alizadeh, A. 2007. Principal of Applied Hydrology. 14rd Edn. Mashhad Emamreza University, 807 pp (In Persion).

2. Blumenfeld, S., C. Lu, T. Christopehersen and D. Coates. 2009. Water, Wetlands and Forests: a Review of Ecological, Economic and Policy Linkages. Secretariat of the Convention on Biological Diversity and Secretariat of the Ramsar Convention on Wetlands, Montreal and Gland. CBD Technical Series, $38 \mathrm{pp}$.

3. Booker, D.J. and T. Snelder. 2012. Comparing Methods for Estimating Flow Duration Curves at Ungauged Sites. Journal of Hydrology, 434: 78-94.

4. Brath, A., A. Castellarin, M. Franchini and G. Galeati. 2001. Estimating the Index Flood Using Indirect Methods. Hydrological Sciences, 46: 399-418.

5. Castellarina, A.G., L. Galeatib, L. Brandimartea, A. Montanaria and A. Bratha. 2004. Regional FlowDuration Curves: Reliability for Ungauged Basins, Advances in Water Resources, 27: 953-965.

6. Cheng, L., M. Yaeger, A. Viglione, E.Ye.S. Coopersmith and M. Sivapalan. 2012. Exploring the Physical Controls of Regional Patterns of Flow Duration Curves - Part 1: Insights from Statistical Analyses, Hydrol. Earth Syst. Science, 16: 4435-4446.

7. Cordova, J.R. and M. Gonzalez. 1997. Sediment Yield in Small Watersheds Based on Stream Flow and Suspended Sediment Discharge Measurements. Soil Technology, 11: 57-65.

8. Costa, V., F. Wilson and M. Naghettini. Fernandez and M. Naghettini. 2014. Regional Models of Flow-Duration Curves of Perennial and Intermittent Streams and Their Use for Calibrating the Parameters of a Rainfall-Runoff Model. Hydrological Sciences Journal, 59: 262-277. 
9. Dario, P., L.V. Noto and F. Viola. 2013. Eco Hydrological Modelling of Flow Duration Curve in Mediterranean River Basins, Advances in Water Resources, 52: 314-327.

10. Eslami, A.R. and A. Shokohi. 2013. Analysis of River Flow, Using Hydrological and Environmental Index, Journal of Watershed Engineering and Management, 5: 125-133 (In Persian).

11. Eslamian, S.S., M. Ghasemi and S. Soltani Gerdefaramarzi. 2012. Computation and Regionalization of Low Flow Indices and Determination of Hydrological Drought Durations in Karkhe Watershed, Journal of Science and Technology of Agriculture and Natural Resources, Water and Soil Science, 59:1-14 (In Persian).

12. Hisdal, H.L., M. Tallaksen, M.B. Clausen, E. Peters and A. Gus-Tard. 2004. Hydrological Drought Characteristics, in: Hydrological Drougth-Processes and Estimation Methods for Stream flow and Groundwater, Edited by: Tallaksen, L.M. and vanLanen, H.A.J., Developments in Water Science, Elsevier Science, 48: 139-198.

13. Iacobellis, V. 2008. Probabilistic Model for the Estimation of T Year Flow Duration Curves, Water Resources Research, 44:1-13.

14. Kazemi, R. and A.R. Eslami. 2013 .Investigation on the Role of Geological Formation and Hydrological Parameter on Base Flow Index, Case Study: Khazar Region, Journal of Watershed Engineering and Management, 5:85-93 (In Persian).

15. Lane, P.N.J., A.E. Best, K. Hickel and L. Zhang. 2005. The Response of Flow Duration Curves to A forestation, Journal of. Hydrology, 310: 253- 265.

16. Lee, S., J. Kim and J.W. Hur. 2013. Assessment of Ecological Flow Rate by Flow Duration and Environmental Management Class in the Geum River, Korea, Environmental Earth Sciences, 68: 1107-1118.

17. Li, M., Q. Shao, L. Zhang and F.H.S. Chiew. 2010. A New Regionalization Approach and its 25 Application to Predict Flow Duration Curve in Ungauged Basins, Journal of Hydrology, 389: 137145.

18. Mohamoud, Y.M. 2008. Prediction of Daily Flow Duration Curves and Stream Flow for Ungauged Catchments Using Regional Flow Duration Curves, Hydrological Sciences, 53: 706-724.

19. Muneepeerakul, R., S. Azaele, G. Botter, A. Rinaldo and I. Rodriguez-Iturbe. 2010. Daily Stream Flow Analysis Based on a Two-Scaled Gamma Pulse Model, Journal of Water Resources Research, 46, W11546.

20. Reed, D.W., D. Jakob. A.J. Robinson D.S. Faulkner and E.J. Stewart. 1999. Regional Frequency Analysis: a New Vocabulary. In: Hydrological Extremes: Understanding, Predicting, Mitigating, Proc IUGG 99 Symposium. Birmingham, IAHS, 255: 237-43.

21. Richards, K.S. 1982. Rivers: form and Process in Alluvial Channels. London: Methuen, 358 pp.

22. Shamaee Zadeh, M. and S. Soltani. 2011. Regional Analysis of Low Flow in North Karoon Basin, Journal of Science and Technology of Agriculture and Resources, Water and Soil Science, 18: 231242 (In Persian).

23. Ward, R.C. and M. Robinson. 2000. Principles of Hydrology, 4th Edition. McGraw-Hill, Berkshire, Eng10 land, $450 \mathrm{pp}$.

24. Westerberg, I.K., J.L. Guerrero, P.M. Younger, K.J. Beven, J. Seibert, S. Halldin, J.E. Freer and C.Y. $\mathrm{Xu}$ 2011. Calibration of Hydrological Models Using Flow-Duration Curves. Hydrology and Earth System Sciences, 15: 2205-2227.

25. Zheng, H., L.C. ZhangLiu, Q. Shao and Y. Fukushima. 2007. Changes in Stream Flow Regime in Headwater Catchments of the Yellow River Basin Since the 1950s. Hydrological Process, 21: 886893. 


\title{
Investigation of the Role of Physiographical and Hydrological Parameters on the Shape of Flow duration Curve (Case Study: Khazar Region)
}

\begin{tabular}{c} 
Rahim Kazemi $^{1}$ and Najaf Gholi Ghiasi ${ }^{2}$ \\
\hline \hline 1- Instructor, Soil Conservation and Watershed Management \\
(Corresponding author: ra_hkazemi@ yahoo.com) \\
2- Assistant Professor, Soil Conservation and Watershed Management \\
Received: April 12, 2015 $\quad$ Accepted: July 27, 2015 \\
\hline
\end{tabular}

\begin{abstract}
The flow duration curve (FDC) is a classical method used to graphically represent the relationship between the frequency and magnitude of stream flow. Several factors are involved in the shape of (FDC), including climatic and physiographic parameter of basin. In this research, the relationship between (FDC) and the physical parameters of the basin were investigated. 20 hydrometric stations with appropriate daily data were selected. Physiographic parameters extracted using the GIS capability and Flow duration curve were plotted using daily flow data. The first and last inflection of the curve was computed, using coding in MATLAB programming environment. Factor analysis was performed and the most important independent factors influencing the form of flow duration curve were identified. The homogeneous analysis based on independent factors, using cluster analysis method was investigated. Finally correlation between the exceedance values at the inflection point and the geometric and hydrologic parameters were investigated. The results showed that the parameters of the perimeter, Area, length of Main River, length of the catchment, the time of concentration, and the average height of the basin were the most important factors affecting the shape of the curve in the study area. All above mentioned parameters has a positive correlation with deformation curve Index. Precipitation was found as the second most influential factor, with a negative correlation.
\end{abstract}

Keywords: Deformation INDEX, Flow Duration Curve, Homogeneous Regions, Inflection, Khazar 